\title{
A Study on the Diversity and Habitat Specificity of Macrofungi of Assam, India
}

\author{
Assma Parveen, Lipika Khataniar, Gunajit Goswami, Dibya Jyoti Hazarika, \\ Pompi Das, Trishnamoni Gautom, Madhumita Barooah and Robin Chandra Boro*
}

\author{
Department of Agricultural Biotechnology, Assam Agricultural University, Jorhat-13, India \\ *Corresponding author
}

\section{A B S T R A C T}

\begin{tabular}{|l|}
\hline Ke y w o r d s \\
Macrofungi, \\
Diversity, Wild- \\
edible, \\
Morphological \\
study, ITS.
\end{tabular}

Studies on the taxonomy and diversity of macrofungi have gained significance during the recent years, as many macrofungi are facing the risk of extinction because of habitat destruction. We report, here a systematic and detailed study on the diversity and distribution of wild mushrooms in the state of Assam, India, based on phenotypic and molecular characteristics. A total 44 samples were collected from various locations of Assam in different seasons during 2013-2015. Out of the total 44 samples, about 18 macrofungi species occurred during summer season, 9 species were recorded during rainy, 13 species were frequently found during autumn, 5 species in winter and 8 species were prominent during spring. The soil was found as major habitat for 18 collected species, while 10 species were collected from the tree/hardwood tree. This study indicated that the diversity and distribution of macrofungi are dependent on the plant community and the environmental conditions. Molecular characterization based on rDNA -ITS- ((Internal transcribed spacer) sequences revealed that the 44 samples belonged to 16 macrofungal families. Out of the 44 samples, 23 samples were reported to be edible and amongst the rest 21 non-edible strains, 5 strains have medicinal properties, 6 strains are poisonous, two has industrial application and the rest have not been fully explored.

\section{Introduction}

Fungi play a significant role in industry, agriculture, medicine, food industry, textiles, and bioremediation (Danielson et al., 1989; Manzi et al., 1999; Krzywinski et al., 2009; Changet al., 2004). Fungi can be broadly classified into three different forms viz., yeast, moulds and macrofungi or mushrooms. Macrofungi are large, conspicuous sporebearing structures and have fleshy, tough umbrella like sporophores that bear holobasidia on the surface of gills or lamellae that hang down from the cap belonging to basidiomycetes and ascomycetes (few under
Zygomycota) (Alexopoulus et al., 2000). Macrofungi are found in varied types of habitats, depending on the composition of tree species and other substrates. The distribution of macrofungal species is low in hot and dry seasons while they are abundant in spring and autumn due to favourable climate and abundance of flora during that time (Pilz et al., 2001). Similar to yeast and moulds, macrofungi also possess immense biological and economic impact since they serve as food, medicine, biocontrol agents and produce of bioactive compounds such as anti-oxidant, 
anti-diabetic, hypocholesterolemic, antitumor, anti-cancer, immunomodulatory, antiallergic, nephroprotective, and anti-microbial agents (Patel et al., 2012). Macrofungi are used in the bioremediation of industrial waste and in the accumulation of heavy metals from the environment (Tuli et al., 2013). There are about 2166 worldwide recognized edible species that include about 470 species possessing medicinal properties. Furthermore, macrofungi are useful indicators of health or age of an ecosystem (Boa et al., 2004).

Assam, situated in the Northeast of India is a constituent unit of the Eastern Himalayan Biodiversity Region; one of the two biodiversity "Hot Spots" in the country. The climatic conditions along with varied physical features in Assam, makes it rich in diversity of ecological habitats such as forests, grasslands, wetlands, which harbour and sustain wide range of floral and faunal species. Assam has a sub-tropical monsoon climate with an average rainfall of around $1,500 \mathrm{~mm}$ per year. The day-time temperature in summer, the rainy season, rises to around $35^{\circ} \mathrm{C}$ and in winter cools to $25^{\circ} \mathrm{C}$ with a night time minimum of around $10^{\circ} \mathrm{C}$ which is favorsthe growth of most of the macrofungal species (Das et al., 2005). Although, macrofungi are good resources for agroindustrial, medicinal (Veena et al., 2012) and commercial purposes, very few studies have reported the macrofungal diversity of Northeast India (Khaund and Joshi, 2014). Several varieties of macrofungi are being consumed by the local tribes of Assam which are harvested from wild habitats but no efforts have been made to cultivate these macrofungi for commercial production. Moreover, there are no scientific reports related to characteristics of the wild edible macrofungi which are slowly disappearing due to habitat loss. Because of these reasons, the farmers and entrepreneurs of Assam have not been able to exploit its agro-economic potential. Therefore, it is crucial to document the diversity, distribution and abundance of these macrofungi in Assam. Such study will not only pave the way towards domestication and commercialization of the wild species for economic benefits but will also help in establishing a strong basis for molecular taxonomy. In this study we aimed to collect macrofungi from different habitats of Assam followed by their taxonomic evaluation using phenotypic and molecular characteristics.

\section{Materials and Methods}

\section{Collection of macrofungi}

Systematic and periodical survey of different forests, tea gardens and other habitats were undertaken during November, 2013 to April, 2015. For this study, the opportunistic sampling of macrofungi protocol, was followed (Mueller et al., 2004). The sampling method involved walking throughout the entire study region and collecting the macrofungi covering up to $80 \%$ of the area. Sampling of the ground-dwelling macrofungi involved careful extraction of the fruit body from the soil and leaf litter, ensuring that the basal surface was intact. The tree-dwelling macrofungi were sampled by cutting off the basidiomata from the trees without disturbing the spore surfaces present below. Different species exhibit different fruiting phenologies, which vary from month to month and at different altitudes and regions.

The survey and collection of mushroom was done from different locations of Assam viz. Nalbari, Barpeta, Jorhat, Sibasagar, Karbi Anglong, Kamrup, Golaghat, Goalpara, Tezpur, Tinsukia, Rangia and Nagaon under different agro-climatic conditions, during the year 2013-2015. The macrofungal samples were collected in different seasons of the year; rainy season (July and August), winter (December and January), spring (March and 
April) and summer (May and June) during 2013-15. Soft macrofungi were collected carefully by using forceps/free hand, while the macrofungi growing on wood were collected along with small part of wood. All collected samples were carefully wrapped in aluminum foil, placed in an air-tight zip-lock bag and labeled with the collection number, location, date, and other data. The photograph of the mushroom was taken in their natural habitat.

\section{Morphological characterization}

Morphological characterization of the macrofungal strains were carried out based on the cap size, cap structure, colour of the fruiting body, dimensions of stipe, gill shape and gill colour as described earlier (Kumar et al., 2015). Further, the fresh sporocarps of mushroom species were cultured to obtain vegetative mycelium from the tissue (small pieces cut from section between the pileus and stipe) using Potato Dextrose Agar (PDA) media and incubated at $25 \pm 2{ }^{\circ} \mathrm{C}$.

Molecular characterization and phylogenetic analysis of the macrofungal strains

For Molecular characterization of the 44 macrofungal isolates were performed by sequencing the rDNA internal transcribed spacer (ITS) region. For DNA extraction, individual strains were cultured on PDA at 25 $\pm 2{ }^{\circ} \mathrm{C}$ for 7 days to obtain mycelia. The genomic DNA was extracted from the pure mycelia using the protocol of Singh et al., (2003). Amplification of the ITS (Internal transcribed spacer) region of the nuclear ribosomal repeat unit was done using universal primer set ITS1 (5'TCCGTAGGTGAACCTGCGG-3') and ITS4 (5'-TCCTCCGCTTATTGATATGC-3') (Thatoi et al., 2014). PCR reaction mixture was prepared as per TaKaRa Ex Taq ${ }^{\circledR}$ DNA polymerase protocol (Takara, Japan) with 20 pmols of each primer, $2 \mathrm{U}$ Taq DNA polymerase and $50 \mathrm{ng}$ genomic DNA in a final volume of $50 \mu \mathrm{L}$ and amplification was performed using a thermal cycler (Applied Biosystems, USA). The PCR program was as follows: initial denaturing step of $94^{\circ} \mathrm{C}$ for 3 min, followed by 35 cycles of denaturation at $94^{\circ} \mathrm{C}$ for $30 \mathrm{sec}$, annealing at $49^{\circ} \mathrm{C}$ for $1 \mathrm{~min}$ and elongation at $72^{\circ} \mathrm{C}$ for $1 \mathrm{~min}$; a final extension step at $72^{\circ} \mathrm{C}$ for $7 \mathrm{~min}$. The amplified products were sequenced as per the BigDye Terminator sequencing protocol in an ABI 377 automated DNA Sequencer (Applied Biosystems, USA). The ITS rDNA sequence reads obtained after sequencing were assembled into contig using CodonCode Aligner (CodonCode Corporation, USA). Sequence similarity tool BLAST was employed to find the similarity of the sequences with known 16S rDNA sequences in the GenBank database and the sequences were deposited in GenBank of NCBI to obtain the accession numbers. Phylogenetic analysis of the isolates was carried out based on ITSrDNA sequences in MEGA6 software (Tamura et al., 2013). A phylogenetic tree was constructed using the ITS-rDNA sequences of the macrofungal strains along with other similar or related macrofungal ITSrDNA sequences retrieved from the NCBI GenBank. The sequences were aligned with Clustal W using default parameters and a neighbor-joining tree was constructed using MEGA6 software (http://www.megasoftware. net/) with Kimura-2 parameter correction and 1000-step bootstrap.

\section{Results and Discussion}

Sample collection and morphological characterization

A total of 44 macrofungal samples were collected from different places of Assam, viz., Nalbari, Barpeta, Jorhat, Sibasagar, 
KarbiAnglong, Kamrup, Golaghat, Goalpara, Tezpur, Tinsukia, Rangia and Nagaon (Fig. 1) during the study period. The details of the sample collection sites are given in Table 1 .

Several workers have studied the diversity of macrofungi from different parts of India namely Kashmir (Sheikh et al., 2014), Garhwal (Vishwakarma et al., 2012), Tamil Nadu (Mani et al., 2009). However very less number of reports appeared from north eastern part of India and only a few attempts have been initiated to document macrofungi from this area. An attempt was made by Gogoi and Sarma to find out some edible macrofungi of Dhemaji district of Assam (Gogoi et al., 2012).

The morphological taxonomic system of classification given by Ainsworth (1963) and modified by Webster (2007) was used for characterization of the macrofungal samples collected from different locations (Table 2). Macroscopic features of the carpophores pertaining to pileus, lamellae, stipe, etc. which play an important role in the taxonomy of macrofungi have been discussed. The photographs of the collected macrofungal samples are shown in Figure 2(a) and 2(b). The fruiting bodies of macrofungi were found to be distinctly different from each other. Some species that grew on wood had caps that attach directly to the wood (e.g. Pycnoporus, Ganoderma). In some cases, the cap was semi-circular and attached by the straight edge while in others the stalk (also called the stipe, or stem) may be central and support the cap in the middle and in Pleurotus, the stalk was off-central or lateral. The morphological data of the collected samples are detailed in Table 2. Similar studies were carried out by Ram et al., (2010) where several edible fleshy fungi were collected from the wilds in Eastern Uttar Pradesh during the rainy season on dead and decaying plant or animal remains. Kalita et al., (2016) documented wild edible macrofungi from Shyrwat and Upper Shillong Reserve Forests of Meghalaya, Northeast India where a total of 22 macrofungi were collected during the rainy season (July to September) 2014, and identified on the basis of macroscopic and microscopic characteristics. The diversity of wild macrofungi in Nagaland, India was reported by Toshinungla et al., (2016) where a total of 87 species of wild macrofungi were collected and identified and out of the collected macrofungi, 37 species were identified as edible, 21 species medicinal, 5 poisonous and 37 inedible/unclassified. Surveys were conducted in Narpuh Reserve Forest, Meghalaya, India to unveil the macrofungal diversity and identified a variety of macrofungi on the basis of macro- and microscopic characteristics.

\section{Seasonal occurrence of the macrofungal species}

Climatic and edaphic conditions prevailing in Assam favoured the occurrence of diverse macrofungi. Our study revealed that 18 species occurred during the summer season, 9 species were recorded during rainy, 13 species were frequently found during autumn, 5 species during winter and 8 species grew mostly in the spring. Comparison of the seasonal occurrence is depicted in Figure 3. Some species were observed to overlap two seasons for e.g. Pleurotus sapidus, Chlorophyllum molybdites were found both in rainy and autumn season; Physisporinus vitreus in both autumn and winter season; Bolbitius titubans and Auricularia sp. grew both during summer and rainy season. Moisture is one of the major factors influencing the fruiting of macrofungi. During the rainy seasons, the availability of adequate moisture favor the growth of the macrofungi. Species diversity was higher in the rainy seasons compared to the early dry seasons, as 
there is adequate moisture available during the rainy seasons. While the macrofungal species collected in the early dry season was predominated by the Polyporales, since there is a decrease in rainfall and relative humidity along with increase in temperature and sunshine, most of the fleshy macrofungi cannot withstand these conditions.

Singh and Prasad (2003) recorded a number of mushroom spp. during 1997-98 and 19981999. The occurrence of species was found to be greater in September during 1997-98 and in August in 1998-99, while July and October exhibited a lesser number of macrofungi. However, in another investigation, July month of each year (2002-05) showed a greater number of macrofungal species. A varying number of macrofungi of different species in different months may be due to low relative humidity, low temperature range, very little and no rainfall.
It was observed that the diversity of the macrofungal species depend on the habitat (Fig. 4). The highest numbers of samples were from Golaghat followed by Nagaon and Goalpara districts of Assam. The soil was found to be the best source of diverse macrofungal species followed by tree and deadwood. A variety of habitats i.e. soil, tree, wood, litter, lawn, etc. supports growth for macrofungi and soil was found as major habitat for 18 collected species, while 10 species were collected from the tree/hardwood tree. The variation observed in occurrence of mushroom species in various habitats may be due to their particular mode of nutrition; the macrofungi growing on the soil are symbiotic, on rotting and dead wood are saprophytic and on trees are parasitic. Cosmopolitan distribution of macrofungi have also been reported (Suryanarayanan et al., 2004), certain species of mushroom are associated with particular kinds of trees and plants (Hawksworth, 2001).

Table.1 GPS data for the macrofungal sample collection sites

\begin{tabular}{|l|l|l|l|}
\hline Sites & Latitude & Longitude & Elevation(m) \\
\hline Mariani & $26^{\circ} 39^{\prime} 26^{\prime \prime} \mathrm{N}$ & $94^{\circ} 18^{\prime} 55^{\prime \prime} \mathrm{E}$ & 118 \\
\hline Goalpara & $26^{\circ} 10^{\prime} 12.8028^{\prime \prime} \mathrm{N}$ & $90^{\circ} 37^{\prime} 35.8212^{\prime \prime} \mathrm{E}$ & 44 \\
\hline Jorhat & $26^{\circ} 45^{\prime} 27 \mathrm{~N}$ & $94^{\circ} 12^{\prime} 11 \mathrm{E}$ & 111 \\
\hline Diphu & $25^{\circ} 50^{\prime} 36^{\prime \prime} \mathrm{N}$ & $93^{\circ} 25^{\prime} 52^{\prime \prime} \mathrm{E}$ & 2000 \\
\hline KarbiAnglong & $26^{\circ} 35^{\prime} \mathrm{N}$ & $93^{\circ} 50^{\prime} \mathrm{E}$ & 186 \\
\hline Sibsagar & $26^{\circ} 59^{\prime} 3 \mathrm{~N}$ & $94^{\circ} 38^{\prime} 16 \mathrm{E}$ & 95 \\
\hline Kamrup & $26^{\circ} 26^{\prime} 57 \mathrm{~N}$ & $91^{\circ} 36^{\prime} 49 \mathrm{E}$ & 38 \\
\hline Golaghat & $26^{\circ} 30^{\prime} 42 \mathrm{~N}$ & $93^{\circ} 57^{\prime} 34 \mathrm{E}$ & 90 \\
\hline Barpeta & $26^{\circ} 19^{\prime} 22 \mathrm{~N}$ & $91^{\circ} 0^{\prime} 23 \mathrm{E}$ & 27 \\
\hline Tezpur & $26^{\circ} 37^{\prime} 60 \mathrm{~N}$ & $92^{\circ} 47^{\prime} 60 \mathrm{E}$ & 47 \\
\hline Nagaon & $26^{\circ} 21^{\prime} 0 \mathrm{~N}$ & $92^{\circ} 40^{\prime} 0 \mathrm{E}$ & 54 \\
\hline Tinsukia & $27^{\circ} 29^{\prime} 30.63^{\prime \prime} \mathrm{N}$ & $95^{\circ} 20^{\prime} 55.30^{\prime \prime} \mathrm{E}$ & 125 \\
\hline Rangia & $26^{\circ} 26^{\prime} 28.61^{\prime \prime} \mathrm{N}$ & $91^{\circ} 37^{\prime} 7.11^{\prime \prime} \mathrm{E}$ & 53 \\
\hline
\end{tabular}


Table.2 Morphological data of the collected macrofungal samples

\begin{tabular}{|c|c|c|c|c|c|c|}
\hline $\begin{array}{l}\text { Sl. } \\
\text { No }\end{array}$ & Samples & $\begin{array}{l}\text { Site of } \\
\text { Collection }\end{array}$ & Habitat & $\begin{array}{l}\text { Fruiting } \\
\text { Body/Colour }\end{array}$ & Stipe & Pileus/Gills \\
\hline 1. & M3 & Mariani & Tree & $\begin{array}{l}\text { Sessile, corky. } \\
\text { Scarlet red to } \\
\text { flame orange }\end{array}$ & $\begin{array}{l}\text { Absent. A small, } \\
\text { single } \\
\text { attachment point }\end{array}$ & $\begin{array}{l}5 \mathrm{~cm} \text { in length. } \\
\text { Pores on the } \\
\text { underside }\end{array}$ \\
\hline 2. & Assam & Mariani & $\begin{array}{l}\text { Hardwood } \\
\text { tree }\end{array}$ & $\begin{array}{l}\text { Flesh is white, } \\
\text { firm, and varies in } \\
\text { thickness. White } \\
\text { to grey or tan to } \\
\text { dark-brown }\end{array}$ & $\begin{array}{c}\text { Absent. Cap } \\
\text { laterally attached }\end{array}$ & $\begin{array}{l}\text { Fan or oyster- } \\
\text { shaped }(11 \mathrm{~cm}) . \\
\text { Decurrent with } \\
\text { white to cream } \\
\text { colour gills }\end{array}$ \\
\hline 3. & T1 & Goalpara & $\begin{array}{l}\text { Hardwood } \\
\text { tree }\end{array}$ & $\begin{array}{l}\text { Woody brackets. } \\
\text { Cream to dark- } \\
\text { brown }\end{array}$ & Absent & $\begin{array}{l}\text { Fan-like or hoof- } \\
\text { like form } \\
\text { honeycomb gills }\end{array}$ \\
\hline 4. & $\mathrm{O}$ & $\begin{array}{l}\text { Titabor, } \\
\text { Jorhat }\end{array}$ & Rice straw & $\begin{array}{l}\text { Flesh is white, } \\
\text { firm, and varies in } \\
\text { thickness }\end{array}$ & $\begin{array}{c}\text { Absent. Cap } \\
\text { laterally attached }\end{array}$ & $\begin{array}{c}\text { Fan or oyster- } \\
\text { shaped }\end{array}$ \\
\hline 5. & Sap & Diphu & Rice straw & $\begin{array}{c}\text { Flesh is white, } \\
\text { firm, and varies in } \\
\text { thickness }\end{array}$ & $\begin{array}{c}\text { Absent. Cap } \\
\text { laterally attached }\end{array}$ & $\begin{array}{l}\text { Fan or oyster- } \\
\text { shaped }(9 \mathrm{~cm}) . \\
\text { Decurrent }\end{array}$ \\
\hline 6. & $\mathrm{CN}$ & AAU, Jorhat & Soil & $\begin{array}{l}\text { Flesh is white, } \\
\text { spongy, and varies } \\
\text { in thickness }\end{array}$ & Absent & $\begin{array}{l}\text { Conical }(8.5 \mathrm{~cm}) \text {. } \\
\text { White to cream in } \\
\text { colour }\end{array}$ \\
\hline 7. & H10 & $\begin{array}{l}\text { AAU, } \\
\text { Jorhat }\end{array}$ & Soil & $\begin{array}{c}\text { Fleshy and varies } \\
\text { in } \\
\text { thickness. Brown }\end{array}$ & Present $(7 \mathrm{~cm})$ & $\begin{array}{l}\text { Depressed }(4 \mathrm{~cm}) \text {. } \\
\text { Extended outwards } \\
\text { (brown in colour) }\end{array}$ \\
\hline 8. & K & $\begin{array}{c}\text { Karbi } \\
\text { Anglong }\end{array}$ & Dead wood & $\begin{array}{c}\text { Tough with a } \\
\text { well-developed } \\
\text { central stalk. Flesh } \\
\text { fibrous. White to } \\
\text { pale brown }\end{array}$ & $\begin{array}{l}\text { Tapering to base } \\
\text { and a persistent } \\
\text { ring is present on } \\
\text { stipe }(4 \mathrm{~cm} \mathrm{x} \\
7 \mathrm{~mm}) \\
\end{array}$ & $\begin{array}{l}\text { Depressed }(7 \mathrm{~cm}) \text {. } \\
\text { Lamellate, (white } \\
\text { to cream in colour) }\end{array}$ \\
\hline 9. & S1 & Goalpara & Tree & $\begin{array}{l}\text { Flesh is white, } \\
\text { firm, and varies in } \\
\text { thickness }\end{array}$ & Tapering to base. & $\begin{array}{l}\text { Fan or oyster- } \\
\text { shaped }(6 \mathrm{~cm}) \\
\text { Decurrent white to } \\
\text { cream in colour }\end{array}$ \\
\hline 10. & $\mathrm{AM}$ & Sibsagar & Soil & $\begin{array}{l}\text { Flesh is cream, } \\
\text { and varies in } \\
\text { thickness. Grey to } \\
\text { pale brown }\end{array}$ & $\begin{array}{l}\text { A persistent ring } \\
\text { is present on } \\
\text { stipe }(7 \mathrm{~cm} \times 20 \\
\mathrm{mm})\end{array}$ & $\begin{array}{l}\text { Flat scales }(4.3 \mathrm{~cm}) \\
\text { serrated, white to } \\
\text { cream in colour }\end{array}$ \\
\hline 11. & $\mathrm{HN}$ & Kamrup & $\begin{array}{l}\text { Hardwood } \\
\text { tree }\end{array}$ & $\begin{array}{l}\text { Corky, hard, } \\
\text { woody- } \\
\text { textured. dark- } \\
\text { brown }\end{array}$ & Absent & $\begin{array}{c}\text { Fan-like or hoof- } \\
\text { like form. } \\
\text { Decurrent (grey to } \\
\text { brown in colour) }\end{array}$ \\
\hline 12. & $\mathrm{~N} 1$ & Diphu & Soil & $\begin{array}{l}\text { Brown spongy } \\
\text { well-developed } \\
\text { central stalk. Flesh } \\
\text { fibrous. }\end{array}$ & Absent & $\begin{array}{l}\text { Radicating, fleshy } \\
\qquad(4.6 \mathrm{~cm})\end{array}$ \\
\hline 13. & $\mathrm{~V}$ & AAU, Jorhat & $\begin{array}{l}\text { Hardwood } \\
\text { tree }\end{array}$ & $\begin{array}{l}\text { Corky, hard, } \\
\text { woody- } \\
\text { textured. dark- } \\
\text { brown } \\
\end{array}$ & Absent & $\begin{array}{l}\text { Kidney shaped (12 } \\
\mathrm{cm}) \text {. Decurrent } \\
\text { (brown in colour) }\end{array}$ \\
\hline
\end{tabular}




\begin{tabular}{|c|c|c|c|c|c|c|}
\hline 14. & $\mathrm{~N} 2$ & Rangia & Soil & $\begin{array}{l}\text { White with shaggy } \\
\text { scales }\end{array}$ & $\begin{array}{l}\text { A loose ring } \\
\text { around the stipe } \\
\text { of } 8 \mathrm{~cm} \text { length }\end{array}$ & $\begin{array}{l}\text { Gills change } \\
\text { rapidly from white } \\
\text { to pink, then to } \\
\text { black. }\end{array}$ \\
\hline 15. & $\mathrm{P} 2$ & Golaghat & Soil & $\begin{array}{l}\text { Short brown blunt } \\
\text { elevated lines on } \\
\text { the lower surface } \\
\text { of the cap and well } \\
\text { developed with } \\
\text { central stalk }\end{array}$ & $\begin{array}{c}\text { Radicating, } \\
\text { fleshy }(4.6 \mathrm{~cm})\end{array}$ & $\begin{array}{c}\text { Depressed (1.3 } \\
\mathrm{cm}) . \text { Decurrent } \\
\text { (brown in colour) }\end{array}$ \\
\hline 16. & $\mathrm{~T} 2$ & Barpeta & Tree & $\begin{array}{l}\text { Blunt elevated } \\
\text { lines on the lower } \\
\text { surface of the cap } \\
\text { without central } \\
\text { stalk }\end{array}$ & Absent & $\begin{array}{c}\text { Depressed (4.3 } \\
\mathrm{cm}) . \text { Free (cream in } \\
\text { colour) }\end{array}$ \\
\hline 17. & H1 & Tezpur & Tree, Wood & $\begin{array}{l}\text { Shiny, dry and } \\
\text { scaly extremely } \\
\text { tough with a well- } \\
\text { developed central } \\
\text { stalk. Whitish to } \\
\text { buff with } \\
\text { cinnamon-brown } \\
\text { scales. }\end{array}$ & $\begin{array}{l}9 \mathrm{~cm} \times 6 \mathrm{~mm} \text {, } \\
\text { with a slight ring } \\
\text { near the apex). }\end{array}$ & $\begin{array}{c}6 \mathrm{~cm} \text { wide, } \\
\text { lamellate gills on } \\
\text { lower surface, } \\
\text { decurrent, edge } \\
\text { sterile, denticulate }\end{array}$ \\
\hline 18. & $\mathrm{C} 1$ & AAU, Jorhat & Soil & $\begin{array}{l}\text { Flesh is cream in } \\
\text { colour, and varies } \\
\text { in thickness. } \\
\text { whitish -brown }\end{array}$ & Absent & $\begin{array}{l}3 \mathrm{~cm} \text { wide, top- } \\
\text { shaped. white to } \\
\text { cream in colour }\end{array}$ \\
\hline 19. & G2 & Nagaon & Soil & $\begin{array}{l}\text { Elevated lines on } \\
\text { the lower surface } \\
\text { of the cap without } \\
\text { central stalk. } \\
\text { Yellowish white }\end{array}$ & $\begin{array}{l}4 \mathrm{~cm} \times 3 \mathrm{~mm}, \\
\text { with a slight ring } \\
\text { near the apex) }\end{array}$ & $\begin{array}{l}\text { Umbonate. Free } \\
\text { (cream in colour) }\end{array}$ \\
\hline 20. & $\mathrm{H} 2$ & Golaghat & Soil & $\begin{array}{c}\text { Blunt elevated } \\
\text { lines on the lower } \\
\text { surface of the cap } \\
\text { with central stalk. } \\
\text { whitish }\end{array}$ & Fleshy $(5.6 \mathrm{~cm})$ & $\begin{array}{c}\text { Depressed }(1.3 \mathrm{~cm}) \\
\text { brown in colour }\end{array}$ \\
\hline 21. & $\mathrm{H} 4$ & Jorhat & Soil & $\begin{array}{l}\text { Blunt elevated } \\
\text { lines on the lower } \\
\text { surface of the cap } \\
\text { without central } \\
\text { stalk }\end{array}$ & $\begin{array}{c}\text { Radicating, } \\
\text { fleshy }(2.3 \mathrm{~cm})\end{array}$ & $\begin{array}{l}\text { Umbonate. Free } \\
\text { (cream in colour) }\end{array}$ \\
\hline 22. & H7 & Golaghat & Soil & $\begin{array}{l}\text { Blunt elevated } \\
\text { lines on the lower } \\
\text { surface of the cap } \\
\text { with central stalk }\end{array}$ & $\begin{array}{l}\text { Tapering to base } \\
\text { and a persistent } \\
\text { ring }\end{array}$ & $\begin{array}{c}\text { Flat }(9.3 \mathrm{~cm}) . \\
\text { (Greyish in colour) }\end{array}$ \\
\hline 23. & H6 & Nagaon & Soil & $\begin{array}{l}\text { Brownish elevated } \\
\text { lines on the lower } \\
\text { surface of the cap } \\
\text { without central } \\
\text { stalk }\end{array}$ & $\begin{array}{l}\text { Tapering to base } \\
\text { and a persistent } \\
\text { ring is present on } \\
\text { stipe }\end{array}$ & $\begin{array}{l}\text { Umbonate. Free } \\
\text { (cream in colour) }\end{array}$ \\
\hline 24. & AP2 & Barpeta & Soil & $\begin{array}{c}\text { a brick-red } \\
\text { colouration in the } \\
\text { center }\end{array}$ & $\begin{array}{l}\text { Light yellow } \\
(4.5-7 \mathrm{~cm} \text { in } \\
\text { diameter })\end{array}$ & $\begin{array}{l}\text { Crowded, } \\
\text { yellowish }\end{array}$ \\
\hline
\end{tabular}




\begin{tabular}{|c|c|c|c|c|c|c|}
\hline 25. & AP3 & Golaghat & Dead tree & $\begin{array}{l}\text { Basidiocarps, } 5 \\
\text { mm thick, waxy } \\
\text { and soft when } \\
\text { fresh, hard and } \\
\text { cartilaginous when } \\
\text { dry }\end{array}$ & $\begin{array}{l}5 \mathrm{~mm} \text { thick, } \\
\text { greyish pore } \\
\text { surface }\end{array}$ & $\begin{array}{c}\text { Pore surface white } \\
\text { to bluish white and } \\
\text { translucent }\end{array}$ \\
\hline 26. & AP5 & Barpeta & rotting wood & $\begin{array}{l}\text { Cream or light } \\
\text { covered in a fine } \\
\text { white bloom } \\
\text { convex, } 4-12 \mathrm{~cm}\end{array}$ & $\begin{array}{c}\text { Centrally } \\
\text { depressed } \\
\text { attached to the } \\
\text { substrate via an } \\
\text { eccentric stem }\end{array}$ & $\begin{array}{c}\text { White; up to } 3.5 \\
\mathrm{~cm} \text { long and } 2.5 \mathrm{~cm} \\
\text { diameter }\end{array}$ \\
\hline 27. & AP6 & Sibsagar & Wood & $\begin{array}{c}\text { Oyster shell- } \\
\text { shaped to fan- } \\
\text { shaped and colour } \\
\text { ranges from ivory } \\
\text { white to pinkish } \\
\text { buff to orange- } \\
\text { grey }\end{array}$ & Absent & $\begin{array}{c}\text { Decurrent, running } \\
\text { a short ways down } \\
\text { the stipe- } 10 \mathrm{~mm} \\
\text { broad, white to } \\
\text { cream in colour }\end{array}$ \\
\hline 28. & AP7 & Sibsagar & Soil & $\begin{array}{c}\text { Pale fawn tan or } \\
\text { pale brownish } \\
\text { towards the centre }\end{array}$ & $\begin{array}{l}\text { Stem } 13 \mathrm{~cm} \mathrm{x} 3- \\
10 \mathrm{~mm} \text {, centric } \\
\text { to excentric }\end{array}$ & $\begin{array}{l}\text { Pileus } 1.5-8 \mathrm{~cm} \\
\text { wide, densely } \\
\text { decurrent gills }\end{array}$ \\
\hline 29. & AP8 & Tinsukia & Soil & $\begin{array}{c}\text { Brownish or } \\
\text { greyish cap } \\
\text { measures } 17 \mathrm{~cm} \text { in } \\
\text { diameter and is } \\
\text { covered with } \\
\text { coarse darker } \\
\text { brown scale }\end{array}$ & $\begin{array}{l}\text { Pale grey or } \\
\text { brown stem ( } 8 \\
\mathrm{~cm}) \text { and }(3.1 \mathrm{in}) \\
\text { high and } 3 \mathrm{~cm} \\
(1.2 \mathrm{in}) \text { wide }\end{array}$ & $\begin{array}{l}\text { The underside } \\
\text { bears soft, pale } \\
\text { grey 'teeth' rather } \\
\text { than gills }\end{array}$ \\
\hline 30. & AP9 & Nagaon & Wood & $\begin{array}{l}\text { Fibrous-corky, } \\
\text { usually } 3-10 \mathrm{~cm} \\
\text { in } \\
\text { diameter,attached } \\
\text { directly to the } \\
\text { substrate } \\
\end{array}$ & Absent & $\begin{array}{l}\text { Soft tomentose, } \\
\text { semi-circular to } \\
\text { elongate Pore } \\
\text { surface pale orange }\end{array}$ \\
\hline 31. & AP11 & Tinsukia & Bark of Tree & $\begin{array}{c}\text { Gelatinous, } \\
\text { orange-yellow } \\
\text { fruit body of the } \\
\text { fungus, which can } \\
\text { grow up to } 4.5 \mathrm{~cm} \\
\text { diameter }\end{array}$ & Absent & $\begin{array}{c}\text { Irregular shape, } \\
\text { and usually breaks } \\
\text { through the bark of } \\
\text { dead branches }\end{array}$ \\
\hline 32. & AP12 & Tinsukia & Lawn & $\begin{array}{c}\text { White or pale } \\
\text { yellowish } \\
\text { developing } \\
\text { pressed-down } \\
\text { fibers or scales } \\
\end{array}$ & $\begin{array}{l}7 \mathrm{~cm} \text { tall, the } \\
\text { parallel stem } \\
\text { with a small } \\
\text { double ringed } \\
\text { bulb at its base }\end{array}$ & $\begin{array}{l}\text { White and finely } \\
\text { scaly, the cap }\end{array}$ \\
\hline 33. & AP13 & Goalpara & Tree & $\begin{array}{l}\text { Basidiocarps } \\
\text { consist of } \\
\text { numerous rosette- } \\
\text { like flattened fan- } \\
\text { shaped pileus }\end{array}$ & Absent & $\begin{array}{l}\text { Cap surface is pale } \\
\text { tan to dull chestnut } \\
\text { brown with finely } \\
\text { fibrillose tiny } \\
\text { scales (squamules) }\end{array}$ \\
\hline 34. & AP14 & Goalpara & Soil & $\begin{array}{l}\text { Cap is } 1.5-5 \mathrm{~cm} \text {, } \\
\text { and grows from } \\
\text { egg-shaped when } \\
\text { young to broadly }\end{array}$ & $\begin{array}{l}\text { 3-10 cm tall and } \\
\text { 2-4 mm wide, } \\
\text { whitish-yellow } \\
\text { colour }\end{array}$ & $\begin{array}{c}\text { Free from the stem } \\
\text { and fragile. } \\
\text { Whitish or pale } \\
\text { yellowish to rusty }\end{array}$ \\
\hline
\end{tabular}


Int.J.Curr.Microbiol.App.Sci (2017) 6(12): 275-297

\begin{tabular}{|c|c|c|c|c|c|c|}
\hline & & & & convex & & cinnamon \\
\hline 35. & AP15 & Golaghat & Forest & $\begin{array}{l}\text { Smaller yellow-to- } \\
\text { lilac-capped } \\
\text { bonnet mushroom }\end{array}$ & $\begin{array}{l}\text { White or pale } \\
\text { pink, smooth } \\
\text { with longitudinal } \\
\text { fibres, with no } \\
\text { stem ring } \\
\end{array}$ & $\begin{array}{l}\text { Pink and crowded, } \\
\text { the broad gills are } \\
\text { deeply sinuate }\end{array}$ \\
\hline 36. & AP16 & Golaghat & Dead wood & $\begin{array}{l}\text { White, thin and } \\
\text { tough scaly with } \\
\text { brown to golden } \\
\text { brown scales and } \\
\text { fibrils }\end{array}$ & $\begin{array}{c}\text { Central or } \\
\text { slightly off- } \\
\text { center; } 2.5 \mathrm{~cm} \\
\text { long; } 3 \mathrm{~mm} \\
\text { wide; brown to } \\
\text { yellowish } \\
\text { brown; scaly to } \\
\text { hairy }\end{array}$ & $\begin{array}{l}\text { Running down the } \\
\text { stem; whitish }\end{array}$ \\
\hline 37. & AP17 & Dergaon & Tree & $\begin{array}{l}\text { Colour of the cap } \\
\text { is purple to } \\
\text { pinkish brown } \\
\text { which is densely } \\
\text { haired }\end{array}$ & $\begin{array}{l}3 \mathrm{~cm} \text { long; } 1 \mathrm{~cm} \\
\text { wide; tough; off- } \\
\text { centre densely } \\
\text { hairy }\end{array}$ & $\begin{array}{l}\text { Running down the } \\
\text { stem; crowded; } \\
\text { purplish. } \\
\text { Gills decurrent }\end{array}$ \\
\hline 38. & $\mathrm{AP} 1$ & Titabar & Dead log & $\begin{array}{l}\text { Yellow to brown } \\
\text { and has scales }\end{array}$ & $\begin{array}{c}\text { The stalk is thick } \\
\text { and short, } 5 \mathrm{~cm} \\
\text { long }\end{array}$ & Gills decurrent \\
\hline 39. & AP19 & Kamrup & Soil & $\begin{array}{c}\text { Orange in colour } \\
\text { and has a calfskin- } \\
\text { like texture }\end{array}$ & $\begin{array}{l}\text { Overlapping } \\
\text { clumps, and } 4 \\
\mathrm{~cm} \text { thick }\end{array}$ & $\begin{array}{l}\text { Wide, shaped like a } \\
\text { fan and attached } \\
\text { direct to the trunk } \\
\text { of a tree }\end{array}$ \\
\hline 40. & AP20 & Kamrup & Soil & $\begin{array}{c}\text { Brown } \\
\text { hygrophanous cap } \\
\text { (striate when } \\
\text { fresh), the disc } \\
\text { usually dingy } \\
\text { yellowish-brown }\end{array}$ & $\begin{array}{l}5 \mathrm{~cm} \text { tall, } 2.5 \\
\text { mm thick, } \\
\text { slender, thin, } \\
\text { fragile }\end{array}$ & $\begin{array}{l}\text { Gills adnexed, } \\
\text { moderately broad }\end{array}$ \\
\hline 41. & AP21 & Jorhat & Litter & $\begin{array}{l}\text { Yellow to brown } \\
\text { with orange caps }\end{array}$ & $\begin{array}{l}8 \mathrm{~cm} \text { long and } 2 \\
\mathrm{~cm} \text { in diameter, } \\
\text { cylindrical; } \\
\text { smooth, with } \\
\text { fine longitudinal } \\
\text { fibres } \\
\end{array}$ & $\begin{array}{c}\text { Adnate; crowded; } \\
\text { yellow }\end{array}$ \\
\hline 42. & $\mathrm{AP} 2$ & Golaghat & Tree & $\begin{array}{l}\text { Flesh is white, } \\
\text { firm, and varies in } \\
\text { thickness. White } \\
\text { to grey or tan to } \\
\text { dark-brown }\end{array}$ & $\begin{array}{l}14 \mathrm{~cm} \text {, convex, } \\
\text { Short and offset } \\
\text { from the centre } \\
\text { of the cap }\end{array}$ & Gills: Decurrent \\
\hline 43. & AP24 & Dergaon & Dead log & $\begin{array}{l}\text { Reminiscent of a } \\
\text { floppy ear, cup- } \\
\text { shaped and has a } \\
\text { tough, gelatinous, } \\
\text { elastic texture }\end{array}$ & $\begin{array}{l}\text { Attached to the } \\
\text { substrate } \\
\text { laterally with } \\
\text { very short stalk }\end{array}$ & Gelatinous gills \\
\hline 44. & AP25 & Nagaon & Lawn & $\begin{array}{l}\text { Brown or beige to } \\
\text { straw colour }\end{array}$ & $\begin{array}{l}6 \mathrm{~cm} \text { tall } \times 2 \mathrm{~mm} \\
\text { thick, equal, } \\
\text { hollow, straw } \\
\text { colour and } \\
\text { annulus absent } \\
\end{array}$ & $\begin{array}{l}\text { Gills are adnate or } \\
\text { adnexed, grey to } \\
\text { purple-brown with } \\
\text { whitish }\end{array}$ \\
\hline
\end{tabular}


Table.3 Nucleotide nblast results of the isolates

\begin{tabular}{|c|c|c|c|}
\hline Sl.No & Samples & Sequencing Result & Accession No. \\
\hline 1. & M3 & Pycnoporus coccineus & KJ862075 \\
\hline 2. & Assam & Pleurotus sapidus & KJ862064 \\
\hline 3. & $\mathrm{~T} 1$ & Ganoderma lucidum & KJ862063 \\
\hline 4. & $\mathrm{O}$ & Pleurotus sapidus & KJ862076 \\
\hline 5. & Sap & Pleurotus ostreatus & KM609393 \\
\hline 6. & $\mathrm{CN}$ & Lycoperdon pyriforme & KM609394 \\
\hline 7. & $\mathrm{H} 10$ & Lactarius friabilis & KM609395 \\
\hline 8. & $\mathrm{~K}$ & Lentinus sajorcaju & KJ862073 \\
\hline 9. & S1 & Pleurotus floridanus & KJ862077 \\
\hline 10. & $\mathrm{AM}$ & Chlorophyllum molybdites & KM609396 \\
\hline 11. & $\mathrm{HN}$ & Ganoderma sp & KM609397 \\
\hline 12. & N1 & Lentinus $s p$ & KM609398 \\
\hline 13. & $\mathrm{~V}$ & Ganoderma applanatum & KM609399 \\
\hline 14. & $\mathrm{~N} 2$ & Coprinus spp. & KM609400 \\
\hline 15. & $\mathrm{P} 2$ & Agrocybe pediades & KM609401 \\
\hline 16. & $\mathrm{~T} 2$ & Ganoderma sp & KM609402 \\
\hline 17. & $\mathrm{H} 1$ & Lentinus critinus & KM609403 \\
\hline 18. & $\mathrm{C} 1$ & Hymenopellis megalospora & KM609404 \\
\hline 19. & $\mathrm{G} 2$ & Leucoagaricus leucothites & KM609405 \\
\hline 20. & $\mathrm{H} 2$ & Agaricus campestris & KM609406 \\
\hline 21. & $\mathrm{H} 4$ & Macrolepiota rhacodes & KM609407 \\
\hline 22. & $\mathrm{H} 7$ & Macrolepiota procera & KM609408 \\
\hline 23. & $\mathrm{H} 6$ & Coprinus disseminatus & KM609409 \\
\hline 24. & AP2 & Hypholomas ublateritium & KR824074 \\
\hline 25. & AP3 & Physisporinus vitreus & KR824075 \\
\hline 26. & AP5 & Pleurotus cornucopiae & KR824077 \\
\hline 27. & AP6 & Pleurotus populinus & KR824078 \\
\hline 28. & AP7 & Panus lecomtei & KR824079 \\
\hline 29. & AP8 & Sarcodon imbricatus & KR824080 \\
\hline 30. & AP9 & Fomitopsiso streiformis & KR824082 \\
\hline 31. & AP11 & Dacrymyces $s p$ & KR824083 \\
\hline 32. & AP12 & Agaricus arvensis & KR824084 \\
\hline 33. & AP13 & Meripilus giganteus & KR824085 \\
\hline 34. & AP14 & Bolbitius titubans & KR824086 \\
\hline 35. & AP15 & Mycena rosea & KR824087 \\
\hline 36. & AP16 & Polyporus arcularius & KR824088 \\
\hline 37. & AP17 & Lentinus strigosus & KR824089 \\
\hline 38. & AP1 & Polyporus squamosus & KR824090 \\
\hline 39. & AP19 & Laetiporus sulphurous & KR824091 \\
\hline 40. & AP20 & Psathyrella corrugis & KR824092 \\
\hline 41. & AP21 & Gymnopilus dilepis & KR824093 \\
\hline 42. & AP2 & Pleurotus pulmonarius & KR824093 \\
\hline 43. & AP24 & Auricularia sp & KR824096 \\
\hline 44. & AP25 & Psilocybe mexicana & KR824097 \\
\hline
\end{tabular}


Table.4 Classification study of macrofungi from different locations of Assam

\begin{tabular}{|c|c|c|c|c|}
\hline ORDER & FAMILY & GENUS & SPECIES & USE \\
\hline \multirow{16}{*}{ Polyporales } & \multirow{9}{*}{ Polyporaceae } & Pycnoporus & coccineus & Industrial \\
\hline & & Lentinus & sajorcaju & Edible \\
\hline & & Lentinus & $s p$ & Edible \\
\hline & & Lentinus & critinus & Edible \\
\hline & & Lentinus & strigosus & Edible \\
\hline & & Laetiporus & sulphurous & Edible \\
\hline & & Panus & lecomtei & Edible \\
\hline & & Polyporus & squamosus & Medicinal \\
\hline & & Polyporus & arcularius & Edible \\
\hline & \multirow[t]{2}{*}{ Meripilaceae } & Physisporinus & vitreus & Industrial \\
\hline & & Meripilus & giganteus & Edible \\
\hline & Fomitopsidaceae & Fomitopsis & ostreiformis & Non-Edible \\
\hline & \multirow{4}{*}{ Ganodermataceae } & Ganoderma & $s p$ & Non-Edible \\
\hline & & Ganoderma & applanatum & Medicinal \\
\hline & & Ganoderma & lucidum & Medicinal \\
\hline & & Ganoderma & $s p$. & Non-Edible \\
\hline \multirow{25}{*}{ Agaricales } & \multirow[t]{7}{*}{ Pleurotaceae } & Pleurotus & Sapidus & Edible \\
\hline & & Pleurotus & Sapidus & Edible \\
\hline & & Pleurotus & ostreatus & Edible \\
\hline & & Pleurotus & floridanus & Edible \\
\hline & & Pleurotus & cornucopiae & Edible \\
\hline & & Pleurotus & pulmonarius & Edible \\
\hline & & Pleurotus & populinus & Edible \\
\hline & \multirow[t]{10}{*}{ Agaricaceae } & Lycoperdon & pyriforme & Edible \\
\hline & & Coprinus & $s p$ & Non-Edible \\
\hline & & Agaricus & arvensis & Edible \\
\hline & & Macrolepiota & rhacodes & Non-Edible* \\
\hline & & Macrolepiota & procera & Non-Edible* \\
\hline & & Chlorophyllum & molybdites & Non-Edible* \\
\hline & & Coprinus & disseminatus & Non-Edible* \\
\hline & & Leucoagaricus & leucothites & Non-Edible \\
\hline & & Agaricus & arvensis & Edible \\
\hline & & Agaricus & campestris & Edible \\
\hline & Bolbitiaceae & Bolbitius & titubans & Non-Edible \\
\hline & Mycenaceae & Mycena & roseae & Non-Edible \\
\hline & \multirow{3}{*}{ Strophariaceae } & Agrocybe & pediades & Non-Edible* \\
\hline & & Psilocybe & mexicana & Non-Edible* \\
\hline & & Hypholoma & sublaterium & Non-edible \\
\hline & Physalacriaceae & Hymenopellis & megalospora & Non-Edible \\
\hline & Cortinariaceae & Gymnopilus & dilepis & Non-Edible \\
\hline & Psathyrellaceae & Psathyrella & corrugis & Non-Edible \\
\hline Russulales & Russulaceae & Lactarius & friabilis & Non-Edible \\
\hline Thelephorales & Bankeraceae & Sarcodon & imbricatus & Edible \\
\hline Dacrymycetales & Dacrymycetaceae & Dacrymyces & $s p$. & Non-Edible \\
\hline Auriculariales & Auriculariaceae & Auricularia & $s p$. & Medicinal \\
\hline
\end{tabular}

* Poisonous macrofungi (Thatoiet al., 2004) 
Fig.1 a) Map of India highlighting Assam b) Satellite image of Assam with different districts of Assam c) Satellite image of Assam showing different sites of collection of macrofungi
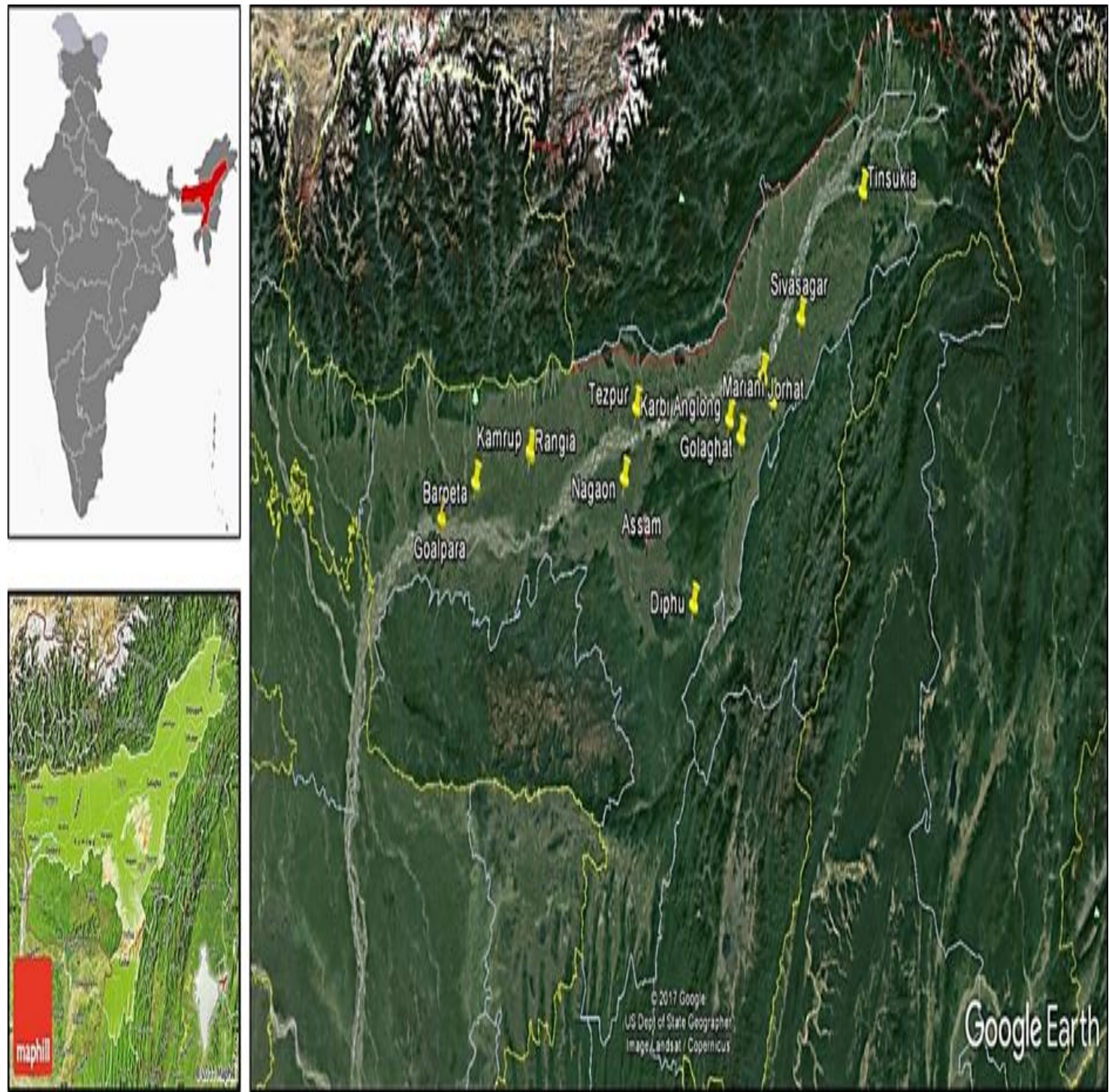
Fig.2 (a) Fruiting bodies of the collected samples. 1= M3, 2=Assam, $3=\mathrm{T} 1$, 4=O, 5= Sap, $6=$ $\mathrm{CN}, 7=\mathrm{H} 10,8=\mathrm{K}, 9=\mathrm{S} 1,10=\mathrm{AM}, 11=\mathrm{HN}, 12=\mathrm{N} 1,13=\mathrm{V}, 14=\mathrm{N} 2,15=\mathrm{P} 2,16=\mathrm{T} 2,17=\mathrm{H} 1,18=$ $\mathrm{C} 1,19=\mathrm{G} 2,20=\mathrm{H} 2,21=\mathrm{H} 4,22=\mathrm{H} 7,23=\mathrm{H} 6,24=\mathrm{AP} 2$

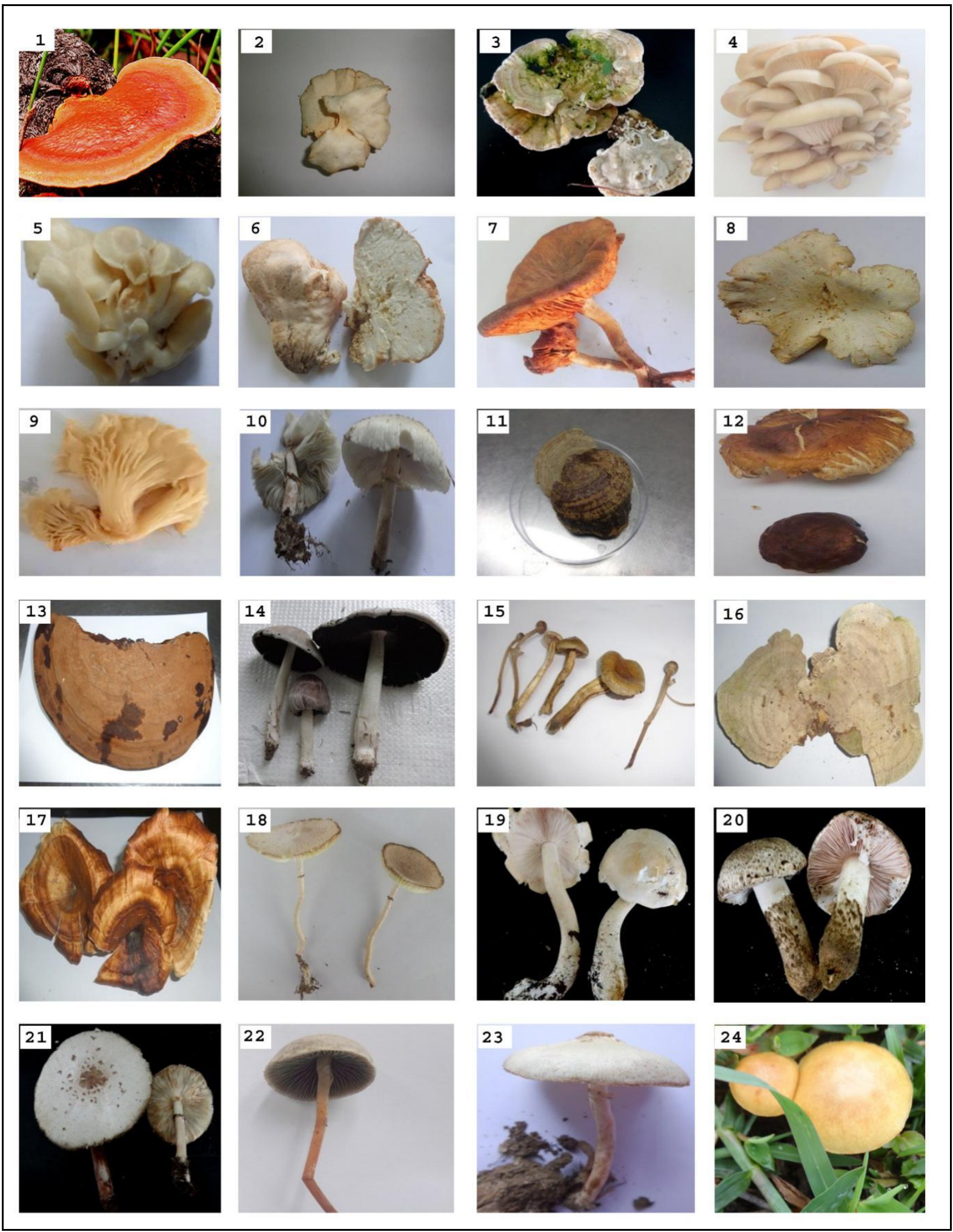


Fig.2 (b) Fruiting bodies of the collected samples. 25= AP3, 26= AP5, 27= AP6, 28= AP7, 29= $\mathrm{AP} 8,30=\mathrm{AP} 9,31=\mathrm{AP} 11,32=\mathrm{AP} 12$, 33= AP13, 34=AP14, 35= AP15, 36= AP16, 37= AP17, $38=\mathrm{AP} 1,39=\mathrm{AP} 19,40=\mathrm{AP} 20,41=\mathrm{AP} 21,42=\mathrm{AP} 2$ 43= AP24, 44=AP25)

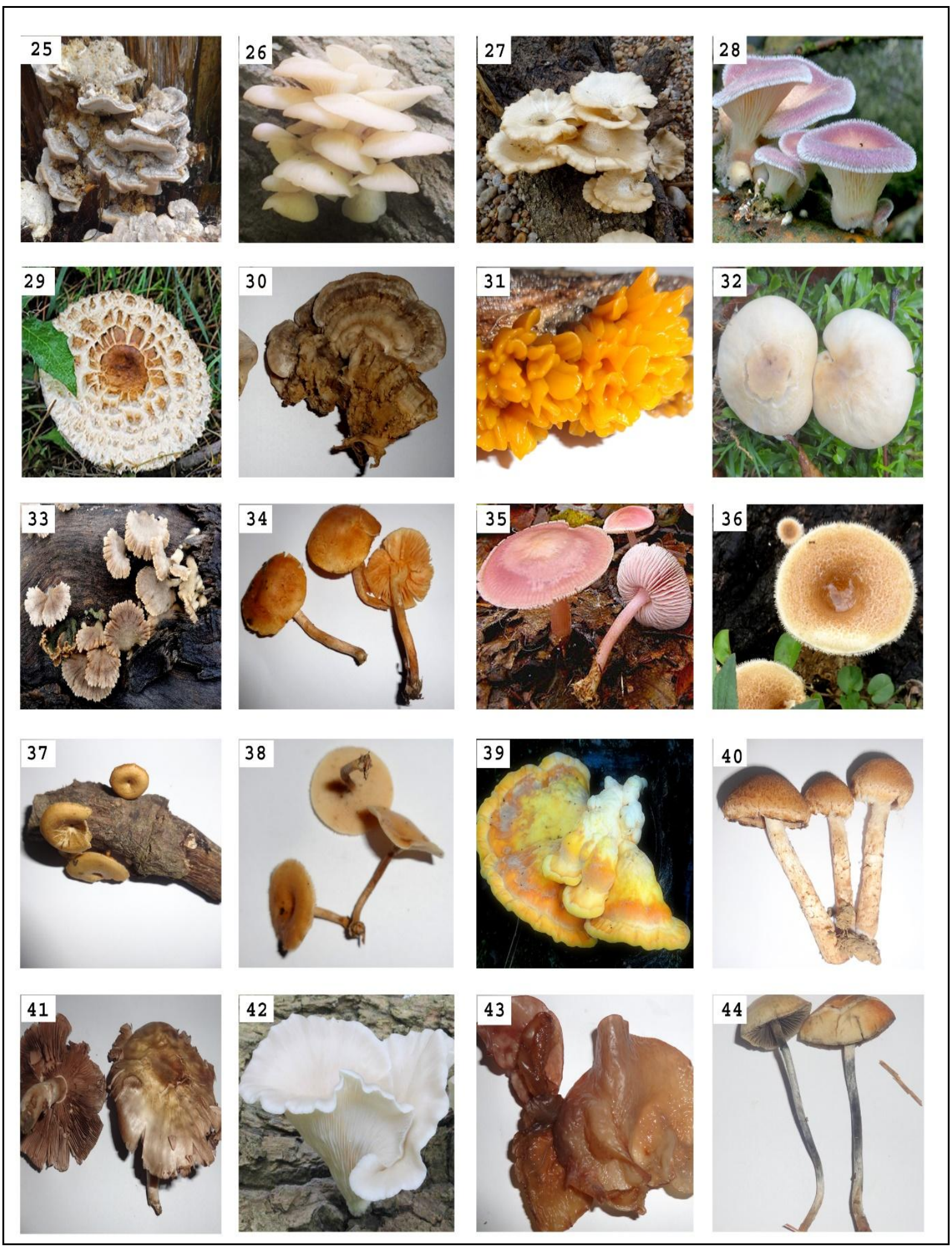


Fig.3 Seasonal distribution of the macrofungal species. Rainy season (July and August), winter (December and January), spring (March and April) and summer (May and June) during 2013-15

Pycnoporus coccineus Pleurotus sapidus Ganoderma lucidum Pleurotus sapidus Pleurotus ostreatus Lycoperdon pyriforme Lactarius friabilis Lentinus sajorcaju Pleurotus floridanus Chlorophyllum molybdites

Ganoderma sp

Lentinus $s p$

Ganoderma applanatum

Coprinus spp.

Agrocybe pediades

Ganoderma sp

Lentinus critinus

Hymenopellis megalospora Leucoagaricus leucothites Agaricus campestris Macrolepiota rhacodes Macrolepiota procera Coprinus disseminatus Hypholomas ublateritium Physisporinus vitreus Pleurotus cornucopiae Pleurotus populinus Panus lecomtei Sarcodon imbricatus Fomitopsiso streiformis Dacrymyces sp Agaricus arvensis Meripilus giganteus Bolbitius titubans Mycena rosea Polyporus arcularius Lentinus strigosus Polyporus squamosus Laetiporussulphurous Psathyrella corrugis Gymnopilus dilepis Pleurotus pulmonarius Auriculariasp Psilocybe mexicana

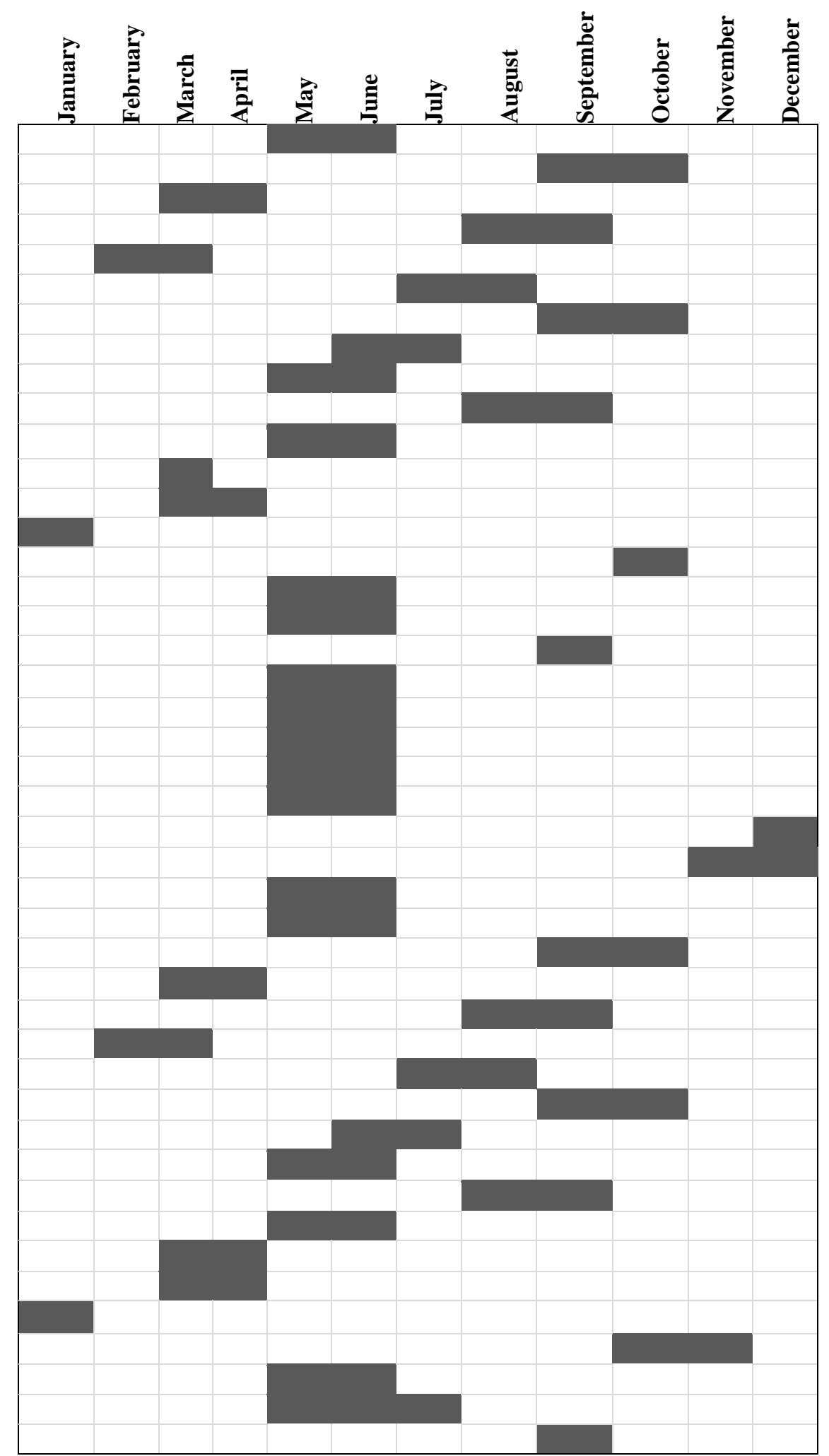


Fig.4 CIRCOS illustration of the samples depicting the relationship between the v macrofungi with that of the site of collection and habitat

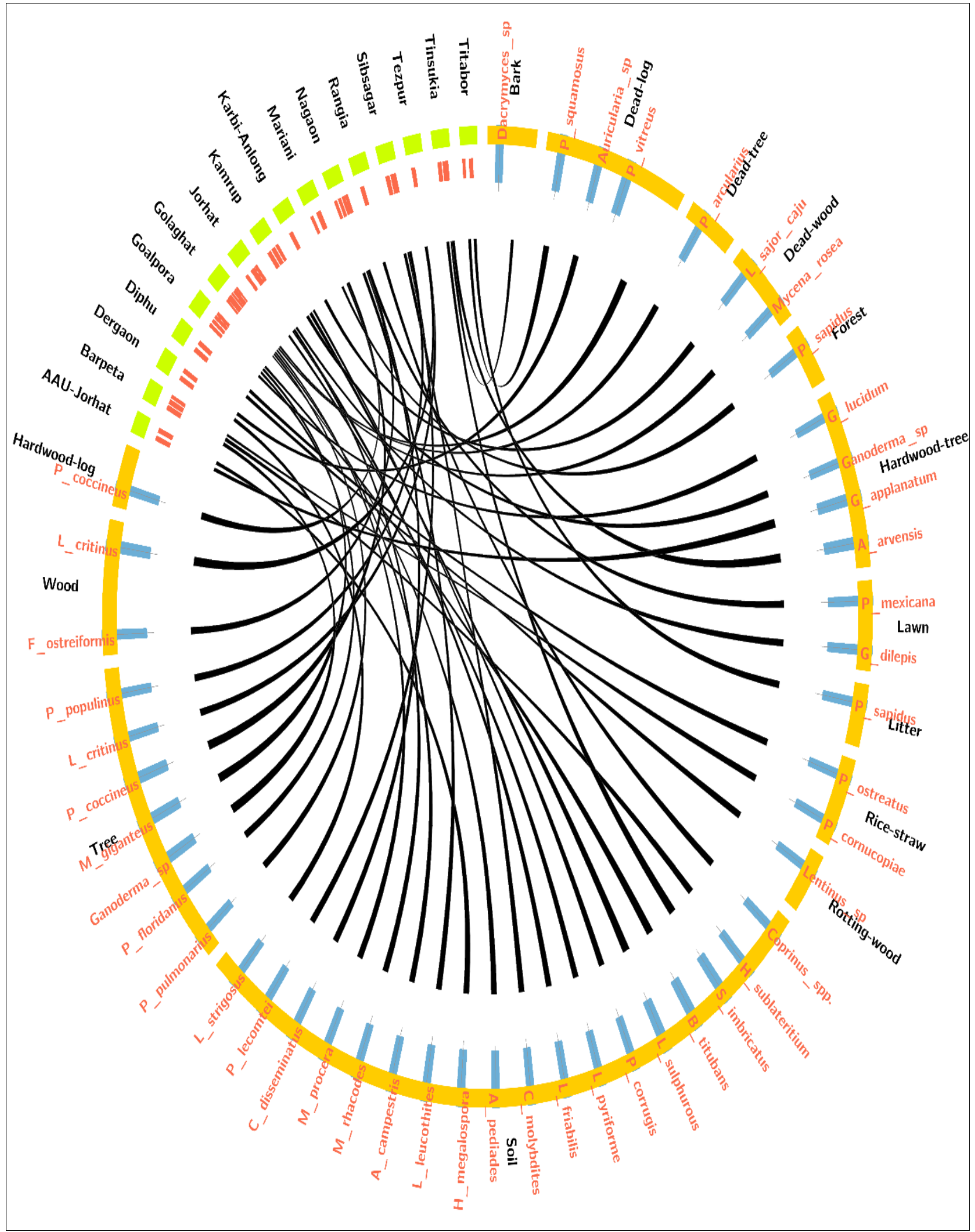


Fig.5 Evolutionary relationships of macrofungal species (NJ Tree Method)

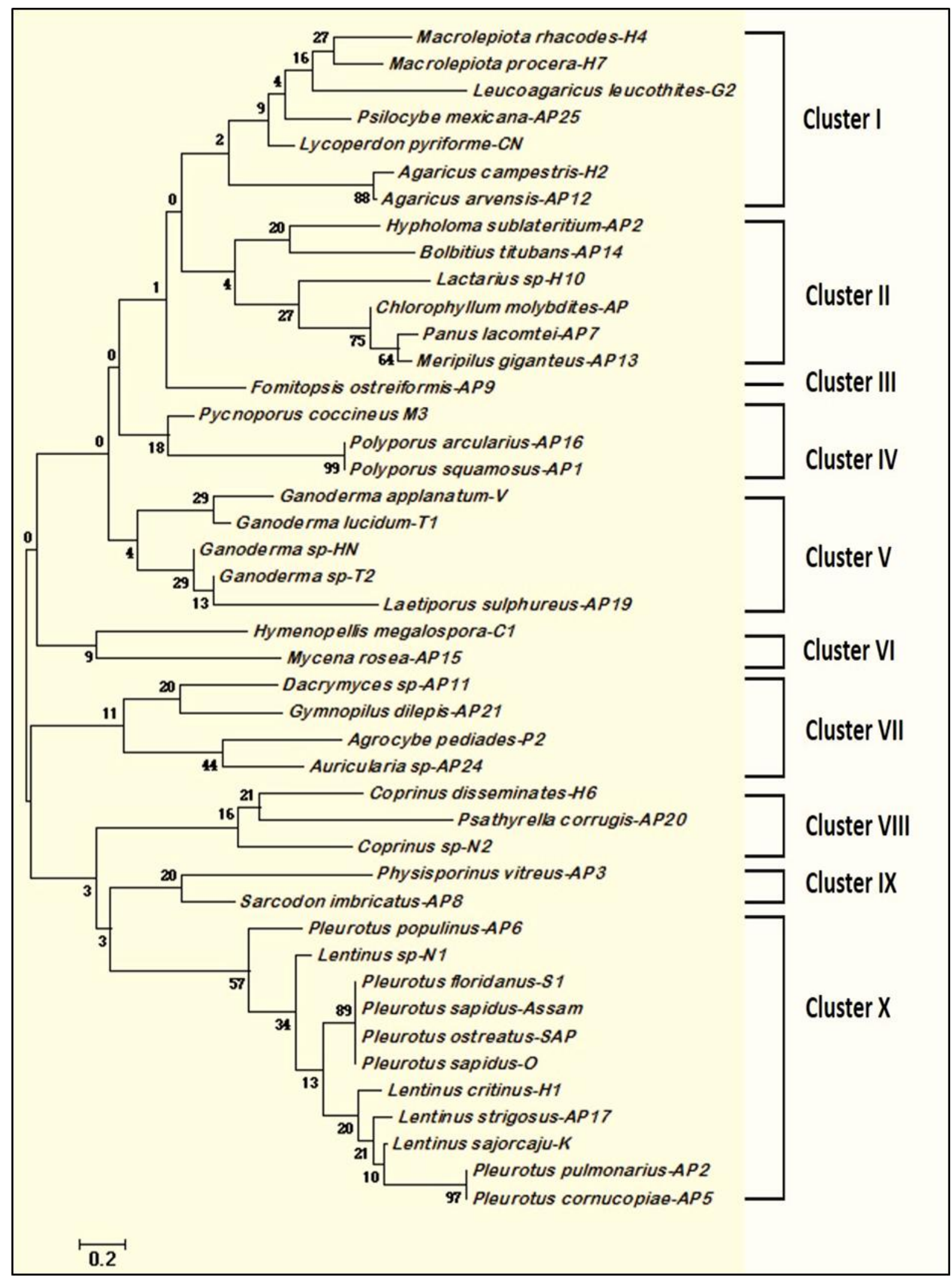


Fig.6 Scientific classification of the identified strains based on molecular data

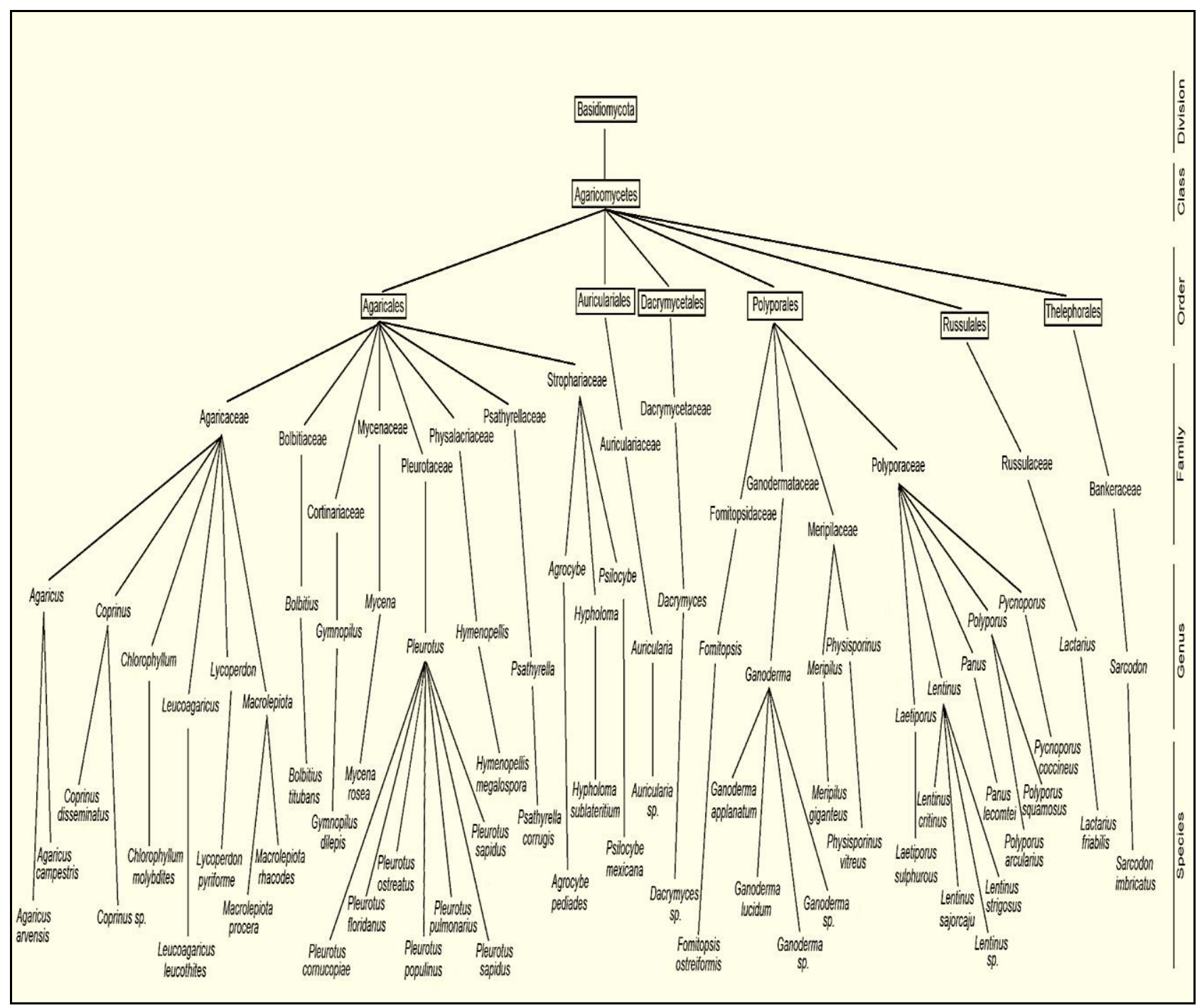

\section{Molecular characterization of the macrofungal strains}

The genomic DNA was subjected to PCRamplification for the ITS region of the rDNA using ITS1 and ITS4 primers. ITS regions of fungal ribosomal RNA are highly variable sequences, which can be useful markers for determining fungal species. The approximate size of the amplified PCR products ( 750bp) was purified and sequenced using ABI 377 automated DNA Sequencer (Applied Biosystems, USA) and resulting contigs were analyzed with CodonCode Aligner version
4.1 (CodonCode Corporation, USA). The consensus sequence obtained for each strain was compared with the ITS rDNA sequences of fungi available in NCBI through a BLAST search and the sequence data has been submitted to GenBank, NCBI and the accession numbers were obtained (Table 3 ). The sequences were aligned with the help of Codoncode Aligner and analysed for homology using nBLAST (NCBI) to obtain possible identities (Altschul et al., 1997). The BLAST results displayed 97-99\% homology with the 44 isolates under study (Table 3). The comparison of ITS gene from different 
strain and different species indicates that there exists high species-specific homogeneity.

Many molecular markers have been applied in phylogenetic studies in order to decipher the relationships among species of Basidiomycetes (Shtaer et al., 2005; Shnyreva and Shtaer, 2006). However, internal transcribed spacer (ITS) I and II the two noncoding polymorphic regions, located between highly conserved regions of $18 \mathrm{~S}, 5.8 \mathrm{~S}$ and 28S rRNA genes (Agarwal et al., 2015), have been widely employed to assess the phylogenetic relationships of mushroom species (Choi et al., 2007). In India, Singh et al., (2006) first reported molecular characterization of mushroom using ITS primers specific to macrofungi and identified 18 mushroom germplasms based on ITS sequence polymorphism. Seven of these isolates were identified as Podaxis pistillaris, six belonged to Phellorinia herculean, four were identified as belonging to Phellinus igniarius and only one was identified as Gymnopilus subearlei. A recent report by Rajaratnam et al., (2012) indicated successful identification of wild macrofungi based on ITS (ITS1 and ITS4) sequence. Their study revealed that the wild mushroom belonged to Agaricomycetes which was a new report of a mushroom in India. Some of the wild edible macrofungi have also been reported from Manipur and Arunachal Pradesh of North East India (Sing et al., 2002). Few strains of Basidiomycetes have also been reported from Assam (Baruah et al., 1971).

Verma et al., (1987) described fleshy fungal flora from Manipur and Meghalaya belonging to the family Auriculariaceae, Clavariaceae, Cantharellaceae, Tricholomataceae, Pluteaceae, Paxillaceae, Cortinariaceae, Cycoperdaceae, and Sclerodermataceae of Basidiomycotina and Halvellaceae of Ascomycotina. Again, Verma et al., (1995) carried out a macrofungal survey of the North
East Hill region of India and confirmed ninety-five species of higher fungi, among these, 85 species were new records. Recently, diversity of wild edible macrofungi from Khasi hills of Meghalaya, India have been reported and a total of 11 different species were identified based on spore morphology (Khaund and Joshi, 2013). Three new species of Russula (Russulasharmae, R. dubdiana and $R$. sikkimensis) from Sikkim (India) have been reported by Das et al., (2013).

\section{Phylogenetic analysis}

Phylogenetic analysis was carried out by comparing the query sequences against a nonredundant database for comparative analysis of ITS partial sequences. The phylogenetic tree generated through the Neighbor-Joining (NJ) method was well supported by the bootstrap values within their nodes. The phylogenetic analysis using partial ITS rRNA gene sequences revealed that the isolate showed closeness with each other. However, further such studies involving more number of isolates (taxa) can better depict their phylogenetic position at a community level. Phylogenetic analysis using the NeighbourJoining tree of the identified strains is shown in Figure 5. The evolutionary history was inferred using the Neighbor-Joining method and the percentage of replicate trees in which the associated taxa clustered together in the bootstrap test (1000 replicates) are shown next to the branches (Efronet al., 1996). The tree is drawn to scale, with branch lengths in the same units as those of the evolutionary distances used to infer the phylogenetic tree. All the 44 macrofungal isolates were grouped into ten clusters (Fig. 5), Cluster I comprised of 7 macrofungal isolates (Macrolepiota rhacodes, Macrolepiota procera, Lycoperdon pyriforme, Leucoagaricus leucothites, Psilocybe Mexicana, Agaricus arvensis, Agaricus campestris), Cluster II comprised of 6 macrofungal isolates (Hypholomas 
ublateritium, Bolbitius titubans, Lactarius sp., Chlorophyllum molybdites, Panuslacomtei, meripiliusgigantius), Cluster III comprised of only 1 macrofungal isolate (Fomitopsiso streiformis), Cluster IV comprised of 3 macrofungal isolates (Pycnoporus coccineus, Polyporus arcularius, Polyporus squamosus), Cluster V comprised of 5 macrofungal isolates (Ganoderma applanatum, Ganoderma lucidum, Ganoderma sp., Laetiporus sulphurous, Ganoderma sp.) Cluster VI comprised of 2 macrofungal isolates (Hymenopellis megalospora, Mycena rosea), Cluster VII comprised of 4 macrofungal isolates (Dacrymyces sp., Gymnopilus dilepis, Agrocybe pediades, Auriculariasp.), Cluster VIII comprised of 3 macrofungal isolates (Coprinus disseminates, Psythrallacorrugis, Coprinus sp.), Cluster IX comprised of 2 macrofungal isolates (Physisporinuscitreus, Sarcodon imbricatus) and Cluster X comprised of 11 macrofungal isolates (Pleurotus populinus, Pleurotus floridanus, Pleurotus sapidus, Pleurotus ostreatus, Pleurotus sapidus, pulmonarius, Pleurotuscornucupaie, Lentinus sp., Lentinus critinus, Lentinus strigosus, Lentinus sajorcaju).

\section{Classification of the macrofungal species}

Classification of the macrofungal species (Table 4) revealed that all the 44 strains belonged to the division Basidiomycota. The macrofungal species can be distributed into 16 families viz. Polyporaceae and Agaricaceae (10 species each), Pleurotaceae (6 species), Ganodermataceae (4 species), Meripilaceae and Strophariaceae (2 species each), Fomitopsidaceae, Russulaceae, Bolbitiaceae, Mycenaceae, Physalacriaceae, Cortinariaceae, Psathyrellaceae, Bankeraceae, Dacrymycetaceae and Auriculariaceae (1 species). The order Agaricales consisting of 23 species was found to be dominant followed by Polyporales with 17 species in this region.
It was observed that, among the 44 samples, 23 strains were edible and among the 21 nonedible strains, 4 strains have medicinal properties, 6 strains are poisonous, one has industrial application and the rest are not fully explored. Singer (1986) recognized 230 genera and 5658 species spread over 17 families in order Agaricales. Previous reports worldwide indicated the edibility and medicinal properties of these species and a complete list of all macrofungal species and countries can be retrieved from www.wildusefulfungi.org (Schwarze et al., 2011).

Scientific classification of the identified strains based on their molecular data has been depicted in Figure 6. The Basidiomycota contains about 30,000 described species, which is $37 \%$ of the described species of true fungi (Kirk et al., 2001). Zhao et al., (2011) provide an overview of recent work on understanding phylogenetic relationships within the Agaricales, a major group within the Agaricomycotina that is the largest clade of mushroom-forming fungi (Matheny et al., 2006).

A timely research regarding isolation, identification and characterization of the existing mushroom flora is essential due to over exploitation and habitat destruction. Thus, our study was an attempt to survey; collect valuable wild macrofungi and their identification based on phenotypic and molecular characteristics, to know the mycotreasure available in this region that is yet to be fully explored. In this study we observed that 23 strains were edible and the rest 21 were non-edible. Among the nonedible strains, 4 strains have medicinal properties, 6 strains are poisonous, one has industrial application and the rest are not fully explored. Further studies on this less explored may provide valuable information as macrofungi have a great potential for the 
production of useful bioactive metabolites and drugs. The list of macrofungi in this study provides the baseline information needed for the identification based on their morphological and molecular characteristics.

\section{Acknowledgements}

Authors are thankful to the Distributed Information Centre, Department of Agricultural Biotechnology, AAU, Jorhat, for providing computational facility and instrumentation facilities to carry out this work. Authors are also grateful to Department of Biotechnology, Govt. of India for providing financial support. Miss Assma Parveen received INSPIRE fellowship from Department of Science and Technology (DST), Govt. of India, and gratefully acknowledges DST.

\section{Conflict of interest}

The authors declare no conflict of interest.

\section{References}

Agrawal, P.K., Agrawal, S and Shrivastava R. 2015. Modern molecular approaches for analyzing microbial diversity from mushroom compost ecosystem. 3 Biotech 5 (6):853-66.

Ainsworth, G.C. Ainsworth \& Bisby's dictionary of the fungi. Cabi; 2008.

Alexopoulus, J.C, Mims, C.W. and Blackwell, M. Intro Myco, 5th edn. National Book Foundation Islamabad, Pakistan, 2000, pp. 508

Altschul, S.F, Madden, T.L., Schäffer, A.A., Zhang, J., Zhang, Z., Miller, W. and Lipman, D.J. 1997. Gapped BLAST and PSI-BLAST: a new generation of protein database search programs. Nucleic acids research 25 (17):3389402.

Avin, F.A., Bhassu, S., Shin, T.Y. and
Sabaratnam, V. 2012. Molecular classification and phylogenetic relationships of selected edible Basidiomycetes species. Molecular Biology Reports 39(7):7355-64.

Baruah, H.K., Singh, D.K. and Islam, M. 1971. On the distribution of higher Basidiomycetes in the Sibsagar district, Assam. Nelumbo 13(3-4):285-9.

Boa, E.R. 2004. Wild edible fungi: a global overview of their use and importance to people. Food \& Agriculture Org.

Choi, H.S., Kim, M.K., Park, H.S., Yun, S.E., Mun, S.P., Kim, J.S., Sapkota, K., Kim, S., Kim, T.Y. and Kim, S.J. 2007. Biological detoxification of lacquer tree (Rhusverniciflua Stokes) stem bark by mushroom species. Food Science and Biotechnology. 16(6):935-42.

Danielson, R.M. and Visser, S. 1989. Host response to inoculation and behaviour of introduced and indigenous ectomycorrhizal fungi of jack pine grown on oil-sands tailings. Canadian Journal of Forest Research 19(11): 1412-21.

Das, K. 2013. Boletus rubripes Thiers, a new record of wild mushroom from Sikkim (India). Taiwania 58(2):136-9.

Efron, B., Halloran, E. and Holmes, S. 1996. Bootstrap confidence levels for phylogenetic trees. Proceedings of the National Academy of Sciences 93(23):13429.

Gogoi, Y. and Sarma, T.C. 2012. An ethnomycological survey in some areas of Dhemaji District (Assam). The Ecoscan 1:403-7.

Hawksworth, D.L. 2001. Mushrooms: the extent of the unexplored potential. International Journal of Medicinal Mushrooms 3(4).

Jonathan, S.G., Okon, C.B, Oyelakin, A.O and Oluranti, O.O. 2012. Nutritional values of oyster mushroom (Pleurotus ostreatus) (Jacq. Fr.) Kumm. Cultivated 
on different agricultural wastes. Nature and Science 10(9):186-91.

Kalita, K., Bezbaroa, R.N., Kumar, R. and Pandey, S. 2016. Documentation of wild edible mushrooms from Meghalaya, Northeast India. Current Research in Environmental \& Applied Mycology. 6(4):238-47.

Khaund, P. and Joshi, S.R. 2014. DNA barcoding of wild edible mushrooms consumed by the ethnic tribes of India. Gene 550(1):123-30.

Krzywinski, M., Schein, J., Birol, I., Connors, J., Gascoyne, R., Horsman, D., Jones, S.J. and Marra, M.A. 2009. Circos: an information aesthetic for comparative genomics. Genome research 19(9): 1639-45.

Kumar, R., Bisht, N.S., Mishra, G., Kalita, K. and Bezbaroa, R. 2015. Micro-and macrofungal diversity in Langol herbal garden Manipur, India. Current Life Sciences 1(1):24-34.

Manzi, P., Aguzzi, A., Vivanti, V., Paci, M. and Pizzoferrato, L. 1999. Mushrooms as a source of functional ingredients. In Proceedings of EURO FOOD CHEMX: 10th European Conference on Functional Foods-A new challenge for the food chemists, Budapest, Hungary (pp. 22-24).

Matheny, P.B., Curtis, J.M., Hofstetter, V., Aime, M.C., Moncalvo, J.M., Ge, Z.W., Yang, Z.L., Slot, J.C., Ammirati, J.F., Baroni, T.J., Bougher, N.L. 2006. Major clades of Agaricales: a multilocus phylogenetic overview. Mycologia 98(6):982-95.

Miles, P.G. and Chang ST. 2004. Mushrooms: cultivation, nutritional value, medicinal effect, and environmental impact. CRC press.

Morton, J.B., Koske, R.E., Stürmer, S.L., Bentivenga, S.P., Mueller, G.M., Bills, G.F. and Foster, M.S. 2004. Mutualistic arbuscular endomycorrhizal fungi.
Biodiversity of Fungi: Inventory and Monitoring Methods 317-36.

Patel, S. and Goyal, A. 2012. Recent developments in mushrooms as anticancer therapeutics: a review. 3 Biotech 2(1):1-5.

Pilz, D. and Molina, R. 2002. Commercial harvests of edible mushrooms from the forests of the Pacific Northwest United States: issues, management, and monitoring for sustainability. Forest Ecology and Management 155(1):3-16.

Rajaratnam, S., Thiagarajan, T. 2012. Molecular characterization of wild mushroom. European Journal of Experimental Biology 2(2):369-73.

Ram, R.C., Pandey, V.N. and Singh, H.B. 2010. Morphological characterization of edible fleshy fungi from different forest regions. Indian Journal of Scientific Research and Technology 1(2):33-5.

Schwarze, F.W. and Schubert, M. 2011. Physisporinus vitreus: a versatile white rot fungus for engineering value-added wood products. Applied Microbiology and Biotechnology 2(3):431-40.

Sheikh, I.A., Vyas, D.E., Ganaie, M.A., Dehariya, K. and Singh, V. 2014. HPLC determination of phenolics and free radical scavenging activity of ethanolic extracts of two polypore mushrooms. International Journal of Pharmacy and Pharmaceutical Sciences 6(2):679-84.

Shnyreva, A.V. and Shtaer, O.V. 2006. Differentiation of closely related oyster fungi Pleurotus pulmonarius and $\mathrm{P}$. ostreatus by mating and molecular markers. Russian Journal of Genetics 42(5):539-45.

Shtaer, O.V., Belokon, Y.S. and Shnyreva, A.V. 2005. Comparative analysis of Pleurotus ostreatus natural isolates. Microbiology 74(2):194-200.

Singer, R. 1986. The Agaricales in modern taxonomy.

Singh, A. and Sharma, S. 2002. Composting 
of a crop residue through treatment with microorganisms and subsequent vermicomposting. Bioresource Technology 85(2):107-11.

Singh, C.S. and Prasad, A.B. 2003. Diversity of Fleshy Fungi in Eastern Uttar Pradesh. Frontiers of Fungal Diversity in India, Int Book dist Co, 327-350 p.327.

Singh, S.K., Sharma, V.P., Sharma, S.R., Kumar, S. and Tiwari, M. 2006. Molecular characterization of Trichoderma taxa causing green mould disease in edible mushrooms. Current Science 427-31.

Singh, S.K., Yadhav, M.C., Upadhyay, R.C., Kamal, S., Rai, R.D. and Tewari, R.P. 2003. Molecular characterization of specialty mushroom germplasm of the National Mushroom Repository. Mushroom Research 12(2).

Suryanarayanan, T.S., Ravishankar, J.P., Venkatesan, G. and Murali, T.S. 2004. Characterization of the melanin pigment of a cosmopolitan fungal endophyte. Mycological research 108(8):974-8.

Swann, E.C., Frieders, E.M. and McLaughlin, D.J. 1999. Microbotryum, Kriegeria and the changing paradigm in basidiomycete classification. Mycologia51-66.

Tamura, K., Peterson, D., Peterson, N., Stecher, G., Nei, M. and Kumar, S. 2011. MEGA5: molecular evolutionary genetics analysis using maximum likelihood, evolutionary distance, and maximum parsimony methods.
Molecular biology and evolution 28(10):2731-9.

Thatoi, H. and Singdevsachan, S.K. 2014. Diversity, nutritional composition and medicinal potential of Indian mushrooms: A review. African Journal of Biotechnology 13(4).

Tuli, H.S., Sandhu, S.S. and Sharma, A.K. 2014. Pharmacological and therapeutic potential of Cordyceps with special reference to Cordycepin. 3 Biotech 4(1):1-2.

Veena, S.S. and Pandey, M. 2012. Medicinal mushrooms-a novel crop for horticultural diversification in India. Indian Journal of Agricultural Sciences 3:8-11.

Verma, R.N., Singh, G.B. and Bilgrami, K.S. 1987. Fleshy fungal flora of NEH IndiaI. Manipur and Meghalaya. Indian Mushroom Science 414-21.

Vishwakarma, M.P., Bhatt, R.P. and Joshi, S. 2012. Macrofungal diversity in moist temperate forests of Garhwal Himalaya. Indian Journal of Science and Technology 5(1):1928-32.

Webster, J. and Weber, R.W.S. 2007. Introduction to fungi 3 ed.

Zhao, R., Karunarathna, S., Raspé, O., Parra, L.A., Guinberteau, J., Moinard, M., De, Kesel A., Barroso, G., Courtecuisse, R., Hyde, K.D. and Guelly, A.K. 2011. Major clades in tropical Agaricus. Fungal Diversity 51(1):279-96.

\section{How to cite this article:}

Assma Parveen, Lipika Khataniar, Gunajit Goswami, Dibya Jyoti Hazarika, Pompi Das, Trishnamoni Gautom, Madhumita Barooah and Robin Chandra Boro. 2017. A Study on the Diversity and Habitat Specificity of Macrofungi of Assam, India. Int.J.Curr.Microbiol.App.Sci. 6(12): 275-297. doi: https://doi.org/10.20546/ijcmas.2017.612.034 